\title{
Infinite-Time Quenching in a Fast Diffusion Equation with Strong Absorption
}

\author{
Michael Winkler
}

\begin{abstract}
This work is concerned with the fast diffusion equation $u_{t}=\Delta u^{m}$ $u^{\kappa}$ in $\mathbb{R}^{n}$, where $0<m<1$ and $\kappa<1$. A global positive solution is said to quench regularly in infinite time if $u\left(x_{k}, t_{k}\right) \rightarrow 0$ for some bounded sequence $\left(x_{k}\right)_{k \in \mathbb{N}}$ and some $t_{k} \rightarrow \infty$, and if $\sup _{(x, t) \in K \times(0, \infty)} u(x, t)<\infty$ for all compact $K \subset \subset \mathbb{R}^{n}$. It is shown that such regular quenching in infinite time occurs for a large class of initial data if $\kappa>m$, whereas it is impossible in one space dimension when $\kappa<-m$ and the solution is radially symmetric and nondecreasing for $x>0$.
\end{abstract}

Mathematics Subject Classification (2000). 35K55, 35K65, 35B40.

Keywords. Fast diffusion, strong absorption, quenching in infinite time.

\section{Introduction}

We consider the Cauchy problem in $\mathbb{R}^{n}$ for the fast diffusion equation with strong absorption,

$$
\begin{cases}u_{t}=\Delta u^{m}-u^{\kappa}, & x \in \mathbb{R}^{n}, \quad t>0, \\ u(x, 0)=u_{0}(x), & x \in \mathbb{R}^{n},\end{cases}
$$

with parameters $0<m<1$ and $\kappa<1$ and positive initial data $u_{0} \in C^{0}\left(\mathbb{R}^{n}\right)$.

It can easily be seen that (1.1) cannot have any bounded positive solution. Namely, a comparison argument involving spatially homogeneous solutions shows that any supposedly global solution of (1.1) with bounded initial data must vanish identically after some finite time and hence undergo a finite-time extinction. There is a considerable literature on parabolic problems with strong absorption that deal with the precise description of the qualitative behavior of solutions near zeroes, addressing a large class of quasilinear and semilinear PDEs of type (1.1) and also including cases where positive values of $u$ are prescribed on the boundary of some bounded domain. Typical questions in this respect concern the time evolution of the support $[2-4,9,12,13]$ as well as quenching rates and profiles $[7,10,14,15]$. 
But also global positive solutions of (1.1), thus existing only for unbounded $u_{0}$, face two possible types of behavior in respect of singularity formation: They can either remain uniformly bounded away from zero and thus ignore the singularity in the absorption term in (1.1); or they approach, though being positive for all times, the value zero somewhere in space as $t \rightarrow \infty$. The occurrence of the latter phenomenon, also referred to as quenching in infinite time, is the objective of the present work. It marks one possibility of infinite-time sigularity formation in diffusion processes, and is thereby closely related to the phenomenon of infinitetime blow-up, aka grow-up, which has been detected to occur in some forced heat equations, and which has received considerable interest within the last decade (see [8] and the references therein).

The study of infinite-time quenching was initiated, to the best of our knowledge, in [19] and studied for the semilinear analogue $u_{t}=\Delta u-u^{\kappa}$ of (1.1) in [6] and [5], both works addressing the associated Dirichlet problem with prescribed boundary values $u=1$ in smooth bounded domains: In [6], it was shown that if the absorption term is strong enough in the sense that $\kappa<-1$ if $n \leq 2$ or $\kappa<-3$ if $n=3$, then quenching in infinite time does not occur in convex domains for solutions which decrease with time. On the other hand, the results in [5] give a complete picture for the choice $u_{0} \equiv 1$ in balls with certain critical radii. It was shown there that then infinite-time quenching occurs if and only if $3 \leq n \leq 9$ and $-\frac{\chi(n)}{2-\chi(n)} \leq \kappa<0$ with $\chi(n)=\frac{n}{2}-\sqrt{n-1}$.

Some results are also known for equations involving nonlinear diffusion; these mainly concentrate on the corresponding Dirichlet problem for the quasilinear degenerate diffusion equation $v_{t}=v^{p} \Delta v-v^{q}$ with positive $p$ and $q<1$, representing the case of slow diffusion. (Observe for comparison that via the transformation $v=u^{m}$, the fast diffusion equation in (1.1) corresponds to an equation of this type with $p=-\frac{1-m}{m}<0$.)

In [21] and [23, Thm. 6.4.2], the possibility of quenching in infinite time was ruled out in the one-dimensional setting for $p>1, q>p-1$ and large domains, and for $p>1, q<p-1$ and arbitrary domains. Moreover, infinite-time quenching is impossible for radially symmetric solutions decreasing with time when $p>1$ and either $n=2$ and $q<p-1$, or $n=3$ and $q<p-3$ [23, Thm. 6.4.3]. On the other hand, quenching in infinite time occurs for $v_{0} \equiv 1$ in certain balls if $1 \leq p \leq 1+\frac{\chi(n)}{2-\chi(n)}$ and $q>p-\frac{\chi(n)}{2-\chi(n)}$ [23, Lemma 6.4.1].

The only result we are aware of that refers to a Cauchy problem also concerns the above slow-diffusion case: In [23, Thm. 5.1.3], it has been shown that regular quenching in infinite time (in the sense of Definition 1.1 below) is impossible when $n=1, p \geq 2$ and $q>2-p$.

To summarize, the previously known results indicate that infinite-time quenching appears to be quite a rarely observed phenomenon in problems involving either linear or slow nonlinear diffusion. In contrast to this, for the fast diffusion problem (1.1) the present work identifies a certain parameter regime and a large set of initial data for which quenching in infinite time occurs. Moreover, 
some qualitative feature of this sigularity formation process will be asserted: The quenching solutions we construct will be free of pathologies such as the conceivable coincidence of quenching points and blow-up points, and thereby comply with the concept of regular quenching introduced as follows.

Definition 1.1. We say that a positive classical solution $u$ of $(1.1)$ in $\mathbb{R}^{n} \times(0, T)$ quenches regularly at time $t=T \leq \infty$ if there exists a bounded sequence $\left(x_{k}\right)_{k \in \mathbb{N}}$ and a sequence of times $t_{k} \rightarrow \infty$ such that $u\left(x_{k}, t_{k}\right) \rightarrow 0$ as $k \rightarrow \infty$, and if

$$
\begin{gathered}
\text { for all } \quad K \subset \subset \mathbb{R}^{n} \quad \text { there exists } C(K)>0 \quad \text { such that } \\
u(x, t) \leq C(K) \text { for all } x \in K \text { and } t \in(0, T) .
\end{gathered}
$$

Using this notion, our main results indicate that regular infinite-time quenching occurs for many (quickly growing) initial data if $\kappa$ is sufficiently large, whereas when $\kappa$ is small, it is impossible for 'any' initial data with arbitrary growth, at least in one space dimension and under additional symmetry and monotonicity hypotheses:

- If $0<m<1$ and $m<\kappa<1$ then the solution of (1.1) quenches regularly in infinite time whenever the initial data $u_{0} \in C^{1}\left(\mathbb{R}^{n}\right)$ grow sufficiently fast as $|x| \rightarrow \infty$ (Theorem 2.9); on the other hand,

- if $n=1,0<m<1$ and $\kappa<-m$ then (1.1) does not possess any radially symmetric solution, nondecreasing for $x>0$, that quenches regularly in infinite time (Theorem 3.4).

Observe that in the neighboring regime $\kappa \geq 1$ of weak absorption, many global positive solutions exist that converge to zero uniformly in space as $t \rightarrow \infty$ : For example, this is the case for all initial data $u_{0}$ satisfying $c_{0} \leq u_{0} \leq c_{1}$ on $\mathbb{R}^{n}$ with positive constants $c_{0}$ and $c_{1}$; namely, by a simple comparison argument, such solutions satisfy $\left[c_{0}^{1-\kappa}+(\kappa-1) t\right]^{-\frac{1}{\kappa-1}} \leq u(x, t) \leq\left[c_{1}^{1-\kappa}+(\kappa-1) t\right]^{-\frac{1}{\kappa-1}}$ if $\kappa>1$ and $c_{0} e^{-t} \leq u(x, t) \leq c_{1} e^{-t}$ when $\kappa=1$. It might therefore not be surprising that those $\kappa<1$ that allow for infinite-time quenching can preferably be found near the value 1 . As to the gap of parameters $\kappa$ between $-m$ and $m$ not captured here, we believe but cannot prove that (regular) quenching in infinite time does not occur as well. As indicated, for instance, by the results in [12] (asserting that quenching takes place everywhere once it occurs at all when $n=1$ and $\kappa \leq-m$ ), it seems that at least on the left boundary $\kappa=-m$ of this gap, quenching properties are similar to those in the regime $\kappa<-m$.

The results concerning $\kappa>m$ are proved in Section 2 , and mainly rely on an interpolation argument (asserting global existence) and a pointwise semi-concavity estimate (ensuring regularity of quenching). Section 3 deals with the case $\kappa<-m$ and is essentially based on some techniques previously used in the analysis blow-up and quenching problems $[7,11,17]$. 


\section{Infinite-time quenching for $\kappa>m$}

Throughout this section we assume that $0<m<\kappa<1$, and that $u_{0}$ is smooth enough such that $u_{0} \in C^{1}\left(\mathbb{R}^{n}\right)$. Of course, the latter assumption is for convenience in notation only and can be relaxed to $u_{0} \in C^{0}\left(\mathbb{R}^{n}\right)$ at the cost of some further technical expense.

The growth restriction under which we shall prove quenching in infinite time is the following quantitative version of the requirement that $u_{0}(x) \rightarrow \infty$ uniformly as $|x| \rightarrow \infty$. Here and in the sequel, by $B_{R}$ we denote the ball in $\mathbb{R}^{n}$ with radius $R$ centered at $x=0$.

(H) There exists a radially symmetric $\varphi \in C^{1}\left(\mathbb{R}^{n}\right)$ with $R \mapsto\|\varphi\|_{L^{\infty}\left(\partial B_{R}\right)}$ nondecreasing such that $u_{0} \geq \varphi$ and

$$
\int_{\mathbb{R}^{n}} \varphi^{-\gamma} \leq M \gamma^{-\mu}
$$

holds for all sufficiently small $\gamma>0$, some $M>1$ and some $\mu<\mu_{0}$, where

$$
\mu_{0}:=\frac{n(\kappa-m)}{2(1-\kappa)} .
$$

Remark. Hypothesis $(\mathrm{H})$ is fulfilled if

$$
u_{0}(x) \geq c_{1} e^{c_{2}|x|^{\beta}} \quad \text { for all } \quad x \in \mathbb{R}^{n}
$$

is valid with some $\beta>\frac{n^{2}}{2} \cdot \frac{\kappa-m}{1-\kappa}$ and positive numbers $c_{1}$ and $c_{2}$.

Remark. For the mere existence proof of a local-in-time solution to (1.1) - up to Corollary 2.3 and even to Lemma $2.5-(\mathrm{H})$ will actually not be needed in this sharp form and may be replaced with the relaxed reqirement that $\inf _{|x|>r} u_{0}(x) \rightarrow \infty$ as $r \rightarrow \infty$.

In order to construct a solution of (1.1), let us fix a decreasing family $\left(u_{0 R}\right)_{R>0}$ of smooth positive functions on $B_{R}$ with $u_{0 R} \rightarrow u_{0}$ in $C_{l o c}^{1}\left(\mathbb{R}^{n}\right)$. We furthermore assume that $\left.u_{0 R}\right|_{\partial B_{R}}=\infty$ in the sense that $u_{0 R}(x) \rightarrow \infty$ uniformly as $|x| \rightarrow R$, that is, we require $\inf _{r<|x|<R} u_{0 R}(x) \rightarrow \infty$ as $r \rightarrow R$. Next, for fixed $R$ we choose an increasing sequence $\left(u_{0 R j}\right)_{j \in \mathbb{N}}$ of smooth positive functions on $\bar{B}_{R}$ such that $u_{0 R j} \rightarrow u_{0 R}$ in $C_{l o c}^{1}\left(B_{R}\right)$ as $j \rightarrow \infty, u_{0 R j} \leq j$ in $B_{R},\left.u_{0 R j}\right|_{\partial B_{R}}=j$ and $\Delta u_{0 R j}^{m}-u_{0 R j}^{\kappa}=0$ on $\partial B_{R}$. (The latter two requirements may be achieved, for instance, by setting $u_{0 R j}(x):=\left(j^{m}-\frac{j^{\kappa}}{2 n}\left(R^{2}-|x|^{2}\right)\right)^{\frac{1}{m}}$ near $|x|=R$.) Then the approximate problems

$$
\left\{\begin{array}{l}
\partial_{t} u_{R j}=\Delta u_{R j}^{m}-u_{R j}^{\kappa}, \quad x \in B_{R}, \quad t>0, \\
u_{R j}(x, t)=j, \quad x \in \partial B_{R}, \quad t>0 \\
u_{R j}(x, 0)=u_{0 R j}(x), \quad x \in B_{R},
\end{array}\right.
$$

have (unique) solutions $u_{R j}$ which exist up to a maximum existence time $T\left(u_{R j}\right) \leq$ $\infty$.

Lemma 2.1. Assume that $(\mathrm{H})$ holds. 
i) For all $\eta>0$ one can find $T_{\min }=T_{\min }(\eta)>0$ such that if $u_{0 R j} \geq \eta$ in $B_{R}$ for some $R>0$ and $j \in \mathbb{N}$ then

$$
u_{R j}(x, t) \geq \frac{\eta}{2} \quad \text { for } \quad|x|<R \quad \text { and } \quad t<T_{\min } .
$$

In particular, $T\left(u_{R j}\right)>T_{\min }\left(\inf _{x \in B_{R}} u_{0 R j}(x)\right)$.

ii) For all $R>0$ there exists $c=c(R)>0$ such that for each $j \in \mathbb{N}$,

$$
u_{R j}(x, t) \geq c \text { for }|x|<R \text { and } t<T\left(u_{R j}\right) .
$$

iii) For all $R>0$ and $\Lambda>0$ one can pick $r \in(0, R)$ and $j_{0} \in \mathbb{N}$ such that for all $j \geq j_{0}$, we have

$$
u_{R j}(x, t) \geq \Lambda \quad \text { for } \quad r<|x|<R \text { and } t<T\left(u_{R j}\right) .
$$

iv) Given $R>0$ and $r \in(0, R)$, there is $c=c(r, R)>0$ such that for all $j \in \mathbb{N}$,

$$
u_{R j}(x, t) \leq c \quad \text { for } \quad|x|<r \quad \text { and } t<T\left(u_{R j}\right) .
$$

Proof. i) This easily results from comparison of $u$ with the 'flat' solution

$$
v(x, t):=\left(\eta^{1-\kappa}-(1-\kappa) t\right)^{\frac{1}{1-\kappa}}
$$

of $v_{t}=\Delta v^{m}-v^{\kappa}$ with initial data $v(\cdot, 0) \equiv \eta$.

ii) Consider $v(x, t):=a\left(1+|x|^{2}\right)^{\frac{1}{m}}$ with $a>0$ small enough such that $v \leq u_{0 R j}$ in $B_{R}$ for all $j$, and such that $a^{\kappa-m} \cdot\left(1+R^{2}\right)^{\frac{\kappa}{m}} \leq 2 n$. Then

$$
\begin{aligned}
v_{t}-\Delta v^{m}+v^{\kappa} & =-2 n a^{m}+a^{\kappa}\left(1+|x|^{2}\right)^{\frac{\kappa}{m}} \\
& \leq a^{m} \cdot\left(-2 n+a^{\kappa-m} \cdot\left(1+R^{2}\right)^{\frac{\kappa}{m}}\right) \\
& \leq 0 \quad \text { in } \quad B_{R} \times(0, \infty),
\end{aligned}
$$

so that the comparison principle yields $u_{R j} \geq a\left(1+|x|^{2}\right)^{\frac{1}{m}} \geq a$.

iii) Given $\Lambda>0$, we fix $\varepsilon>0$ small such that $(2 \varepsilon)^{-\alpha} \geq \Lambda$, where $\alpha:=\frac{2}{\kappa-m}$ is positive since $\kappa>m$. It is easy to see that there exists some large $A_{0}>0$ such that

$$
2 m \alpha A_{0}^{2}\left[n\left(R^{2}-\rho^{2}\right)+2(m \alpha+1) \rho^{2}\right] \geq 1 \quad \text { for all } \rho \in[0, R] .
$$

Since $u_{0 R}(x) \rightarrow \infty$ uniformly as $|x| \rightarrow R, u_{0 R}^{-\frac{1}{\alpha}}$ is continuous in $\bar{B}_{R}$ with $\left.u_{0 R}^{-\frac{1}{\alpha}}\right|_{\partial B_{R}}=$ 0 . We thus can find $A \geq A_{0}$ fulfilling

$$
u_{0 R}^{-\frac{1}{\alpha}}(x) \leq A\left(R^{2}-|x|^{2}\right)+\frac{\varepsilon}{2} \quad \text { for all } \quad x \in B_{R} .
$$

By Dini's theorem, the convergence $u_{0 R j}^{-\frac{1}{\alpha}} \rightarrow u_{0 R}^{-\frac{1}{\alpha}}$ is uniform on $\bar{B}_{R}$, hence for some $j_{0} \in \mathbb{N}$ we have

$$
u_{0 R j}^{-\frac{1}{\alpha}}(x) \leq A\left(R^{2}-|x|^{2}\right)+\varepsilon \quad \text { for all } \quad x \in B_{R} \quad \text { and } \quad j \geq j_{0},
$$

so that for such $j$,

$$
v(x, t):=\left(A\left(R^{2}-|x|^{2}\right)+\varepsilon\right)^{-\alpha}, \quad(x, t) \in B_{R} \times(0, \infty),
$$


lies below $u_{R j}$ at $t=0$ and on $\partial B_{R}$. Since furthermore the choices of $\alpha$ and $A$ imply

$$
\begin{aligned}
v_{t}-\Delta v^{m}+v^{\kappa}= & \left(A\left(R^{2}-|x|^{2}\right)+\varepsilon\right)^{-\kappa \alpha} \\
& \cdot\left\{-2 m \alpha A^{2}\left[n\left(R^{2}-|x|^{2}\right)+2(m \alpha+1)|x|^{2}\right]-2 n m \alpha A \varepsilon+1\right\} \\
\leq & 0 \text { in } B_{R} \times(0, \infty),
\end{aligned}
$$

we obtain $u_{R j} \geq v$ in $B_{R} \times(0, \infty)$ by comparison. With $r:=\sqrt{R^{2}-\frac{\varepsilon}{A}}$, for any $x$ with $r<|x|<R$ and all $t>0$ in particular we have

$$
\begin{aligned}
u_{R j}(x, t) & \geq v(x, t) \\
& \geq(2 \varepsilon)^{-\alpha} \geq \Lambda .
\end{aligned}
$$

iv) Let $R_{0}:=\frac{R+r}{2}$ and $a_{0}>0$ be small such that

$$
2 m \alpha R_{0}^{2} a_{0}^{2}[n+2(m \alpha+1)] \leq 1,
$$

where again $\alpha=\frac{2}{\kappa-m}$. Since $u_{0 R}$ is bounded in $\bar{B}_{R_{0}}$, there is $a \leq a_{0}$ such that for $x \in B_{R_{0}}$ we have $u_{0 R}(x) \leq\left(a\left(R_{0}^{2}-|x|^{2}\right)\right)^{-\alpha}$ and therefore $u_{R j}$ is majorized by

$$
v(x, t):=\left(a\left(R_{0}^{2}-|x|^{2}\right)\right)^{-\alpha}
$$

on the parabolic boundary of $B_{R_{0}} \times\left(0, T\left(u_{R j}\right)\right)$. As

$$
\begin{aligned}
v_{t}-\Delta v^{m}+v^{\kappa} & =\left(a\left(R_{0}^{2}-|x|^{2}\right)\right)^{-\kappa \alpha} \cdot\left\{-2 m \alpha a^{2}\left[n\left(R_{0}^{2}-|x|^{2}\right)+2(m \alpha+1)|x|^{2}\right]+1\right\} \\
& \geq\left(a\left(R_{0}^{2}-|x|^{2}\right)\right)^{-\kappa \alpha} \cdot\left\{-2 m \alpha a^{2}\left[n R_{0}^{2}+2(m \alpha+1) R_{0}^{2}\right]+1\right\} \\
& \geq 0 \quad \text { in } B_{R_{0}} \times(0, \infty)
\end{aligned}
$$

by assumption on $a$, the comparison principle yields $u_{R j} \leq v$ for all $j$, which implies the claim, because $v$ is uniformly bounded in $B_{r} \times(0, \infty)$.

Since the $u_{R j}$ are ordered by the comparison principle, from Lemma 2.1 and parabolic Schauder estimates we immediately obtain that the $u_{R j}$ converge to a classical solution of a boundary value problem with boundary data $+\infty$ on $\partial B_{R}$ :

Corollary 2.2. The functions $u_{R j}$ exist globally (that is, $T\left(u_{R j}\right)=\infty$ ) and there exists a positive function $u_{R}$ such that

$$
u_{R j} \nearrow u_{R} \quad \text { in } B_{R} \times(0, \infty) \quad \text { as } j \rightarrow \infty
$$

holds, the convergence taking place in the topology of $C^{0}\left(B_{R} \times[0, \infty)\right) \cap C_{\text {loc }}^{\infty}\left(B_{R} \times\right.$ $(0, \infty))$. The function $u_{R}$ satisfies

$$
\left\{\begin{array}{l}
\partial_{t} u_{R}=\Delta u_{R}^{m}-u_{R}^{\kappa}, \quad x \in B_{R}, \quad t>0, \\
u_{R}(x, t)=\infty, \quad x \in \partial B_{R}, \quad t>0, \\
u_{R}(x, 0)=u_{0 R}, \quad x \in B_{R},
\end{array}\right.
$$


where the second condition is fulfilled in the sense that

$$
\inf _{r<|x|<R, t>0} u_{R}(x, t) \rightarrow \infty \quad \text { as } \quad r \rightarrow R .
$$

Again by comparison, we find that also the family $\left(u_{R}\right)_{R>0}$ is ordered and thus decreases to some limit. More precisely:

Corollary 2.3. We have

$$
u_{R} \searrow u \text { in } \mathbb{R}^{n} \times(0, \infty) \text { as } R \nearrow \infty
$$

for some nonnegative function $u: \mathbb{R}^{n} \times(0, \infty) \rightarrow \mathbb{R}$. Moreover, there exists a maximal $T_{\max } \in(0, \infty]$ such that $u$ is positive on $\mathbb{R}^{n} \times\left(0, T_{\max }\right)$, the convergence $u_{R} \rightarrow u$ takes place in $C_{\text {loc }}^{0}\left(\mathbb{R}^{n} \times\left[0, T_{\max }\right)\right) \cap C_{\text {loc }}^{\infty}\left(\mathbb{R}^{n} \times\left(0, T_{\max }\right)\right)$ and $u$ satisfies (1.1) in $\mathbb{R}^{n} \times\left(0, T_{\max }\right)$. Finally, $u$ is locally bounded from above in $\mathbb{R}^{n}$, uniformly with respect to $t \in\left(0, T_{\max }\right)$.

Proof. As $u_{0}$ is strictly positive in $\mathbb{R}^{n}$ by assumption, we can apply Lemma 2.1 i) to see that $T_{\max }$ indeed is positive. Boundedness from above is a consequence of Lemma $2.1 \mathrm{iv}$ ) and the monotone convergence. The rest immediately follows from parabolic Schauder theory.

The next lemma provides some kind of semi-concavity estimate that has a large number of parallels in the theory of quasilinear parabolic equations, but also of Hamilton-Jacobi equations [1, 16,22].

Lemma 2.4. For all $j \in \mathbb{N}$ and $R>0$, we have

$$
\frac{\partial_{t} u_{R j}}{u_{R j}} \leq \frac{1}{(1-m) t} \quad \text { in } \quad B_{R} \times(0, \infty) .
$$

Consequently,

$$
\frac{u_{t}}{u} \leq \frac{1}{(1-m) t} \quad \text { in } \quad \mathbb{R}^{n} \times(0, \infty)
$$

Proof. The function $z:=\frac{\partial_{t} u_{R j}^{m}}{u_{R j}^{m}}$ is continuous in $\bar{B}_{R} \times[0, \infty)$ and satisfies

$$
z_{t}=-\frac{1-m}{m} z^{2}-(\kappa-m) u^{-1+\kappa} z+m u^{-1}\left(u^{m} \Delta z+2 \nabla u^{m} \cdot \nabla z\right)
$$

in $B_{R} \times(0, \infty)$. Since $z=0$ on $\partial B_{R}$ and $\frac{\kappa-m}{m}$ is positive, the comparison principle tells us that $z$ lies below the (spatially homogeneous) solution $y(t)$ of $y^{\prime}(t)=$ $-\frac{1-m}{m} y^{2}(t)$ for $t>0$ with $y(0)=\infty$, that is, $z \equiv \frac{m \partial_{t} u_{R j}}{u_{R j}} \leq \frac{m}{(1-m) t}$.

As a consequence, we infer that once a solution of (1.1) becomes small, it must do so in a comparatively large subset of $\mathbb{R}^{n}$.

Lemma 2.5. Suppose $u$ is radially symmetric and nondecreasing with respect to $|x|$. Then for all $\tau>0$ there exists $c_{1}>0$ such that if $u(0, t) \leq 1$ for some $t \geq \tau$ then $u(x, t) \leq 2 u(0, t)$ whenever $|x| \leq c_{1} u^{-\frac{\kappa-m}{2 m}}(0, t)$. 
Proof. We fix $t \geq \tau$ and set $v(r):=u^{m}(x, t)$ for $r=|x|$, and $v_{0}:=u^{m}(0, t)$. We assume that $v_{0} \leq 1$, and that for some $r_{0}>0, v(r)<2^{m} v_{0}$ for $r<r_{0}$ and $v\left(r_{0}\right)=2^{m} v_{0}$. From Lemma 2.4 we know that for $r \in\left(0, r_{0}\right)$

$$
\begin{aligned}
v_{r r}+\frac{n-1}{r} v_{r} & \leq \frac{1}{(1-m) t} v^{\frac{1}{m}}+v^{\frac{\kappa}{m}} \\
& \leq\left(\frac{2^{1-\kappa}}{(1-m) \tau}+1\right) \cdot v^{\frac{\kappa}{m}} \\
& =: c_{2} v^{\frac{\kappa}{m}} .
\end{aligned}
$$

Multiplying this by $v_{r} \geq 0$, we obtain

$$
\begin{aligned}
\frac{1}{2} v_{r}^{2}(r) & \leq \frac{c_{2} m}{\kappa+m}\left(v^{\frac{\kappa+m}{m}}(r)-v_{0}^{\frac{\kappa+m}{m}}\right) \\
& \leq \frac{c_{2} m}{\kappa+m} v^{\frac{\kappa+m}{m}}(r) \text { for all } r \in\left(0, r_{0}\right)
\end{aligned}
$$

and therefore upon integration

$$
v(r) \leq v_{0} \cdot\left(1-c_{3} v_{0}^{\frac{\kappa-m}{2 m}} r\right)^{-\frac{2 m}{\kappa-m}} \quad \text { for all } \quad r \in\left(0, r_{0}\right)
$$

with $c_{3}:=\frac{\kappa-m}{2 m} \sqrt{\frac{2 c_{2} m}{\kappa+m}}$. Thus, if

$$
r<\frac{1-2^{-\frac{\kappa-m}{2}}}{c_{3}} v_{0}^{-\frac{\kappa-m}{2 m}}
$$

then we necessarily have $r<r_{0}$ and $v(r)<2^{m} v_{0}$.

With the aid of the last assertion we can exclude that under condition $(\mathrm{H})$ our solution quenches in finite time.

Lemma 2.6. Suppose $(\mathrm{H})$ holds. Then $u$ is positive in $\mathbb{R}^{n} \times[0, \infty)$ and hence a global classical solution of (1.1). Moreover, u satisfies the estimate

$$
u(x, t) \geq c t^{-\frac{\mu m}{\left(\mu_{0}-\mu\right)(1-\kappa)}} \quad \text { for all } x \in \mathbb{R}^{n} \text { and any } t>0 .
$$

In the proof of Lemma 2.6 two more lemmas are required. The first one is an adaptation of the classical Gagliardo-Nirenberg inequality (cf. [20]) to our situation. For the proof of Lemma 2.6 it is important to notice that the appearing constant can be chosen to be independent of the (small) summability power in the least order term on the right hand side of the estimate.

Lemma 2.7. Suppose $r_{0} \in(0,2]$. Then there is a constant $C_{G N}=C_{G N}\left(n, r_{0}\right)>0$ such that for all $r \in\left[r_{0}, 2\right]$, any $s \in(0, \min \{1, r\})$ and all $\psi \in L^{s}\left(\mathbb{R}^{n}\right)$ with $\nabla \psi \in L^{2}\left(\mathbb{R}^{n}\right)$, the estimate

$$
\|\psi\|_{L^{r}\left(\mathbb{R}^{n}\right)} \leq C_{G N}\|\nabla \psi\|_{L^{2}\left(\mathbb{R}^{n}\right)}^{a}\|\psi\|_{L^{s}\left(\mathbb{R}^{n}\right)}^{1-a}
$$

holds, where the number $a \in(0,1)$ is defined by

$$
-\frac{n}{r}=\left(1-\frac{n}{2}\right) a-\frac{n}{s}(1-a) .
$$


Proof. As $s<r \leq 2$ and $s<1<2$, Hölder's inequality gives

$$
\|\psi\|_{L^{r}\left(\mathbb{R}^{n}\right)} \leq\|\psi\|_{L^{2}\left(\mathbb{R}^{n}\right)}^{b}\|\psi\|_{L^{s}\left(\mathbb{R}^{n}\right)}^{1-b} \quad \text { with } \quad b=\frac{2(r-s)}{r(2-s)}
$$

and

$$
\|\psi\|_{L^{1}\left(\mathbb{R}^{n}\right)} \leq\|\psi\|_{L^{2}\left(\mathbb{R}^{n}\right)}^{c}\|\psi\|_{L^{s}\left(\mathbb{R}^{n}\right)}^{1-c} \quad \text { with } \quad c=\frac{2(1-s)}{2-s} .
$$

Using the standard Gagliardo-Nirenberg inequality (cf. [20, Chapter 3.4]), we infer that

$$
\|\psi\|_{L^{2}\left(\mathbb{R}^{n}\right)} \leq c_{1}\|\nabla \psi\|_{L^{2}\left(\mathbb{R}^{n}\right)}^{d}\|\psi\|_{L^{1}\left(\mathbb{R}^{n}\right)}^{1-d}, \quad \text { where } \quad d=\frac{n}{n+2} .
$$

Combining these relations, we obtain

$$
\|\psi\|_{L^{r}\left(\mathbb{R}^{n}\right)} \leq c_{1}^{\frac{b}{1-(1-d) c}}\|\nabla \psi\|_{L^{2}\left(\mathbb{R}^{n}\right)}^{\frac{b d}{1-(1-d) c}}\|\psi\|_{L^{s}\left(\mathbb{R}^{n}\right)}^{1-b+\frac{b(1-c)(1-d)}{1-(1-d) c}} .
$$

As $\frac{1-s}{2-s} \leq \frac{1}{2}$, we estimate

$$
\begin{aligned}
\frac{b}{1-(1-d) c} & =\frac{2}{r} \frac{r-s}{2-s}\left(1-\frac{4}{n+2} \frac{1-s}{2-s}\right)^{-1} \\
& \leq \frac{2}{r_{0}} \frac{n+2}{n}
\end{aligned}
$$

hence the constant in (2.6) is independent of $r \in\left[r_{0}, 2\right]$ and $s \in(0, \min \{1, r\})$. Now an elementary calculation shows that $\frac{b d}{1-(1-d) c}$ coincides with $a$ and thus $(2.4)$ follows.

We shall also need the following elementary variant of Gronwall's lemma.

Lemma 2.8. Let $T>0$ and suppose $y \in C^{0}([0, T])$ satisfies

$$
y(t) \leq y_{0}+c_{0} \int_{0}^{t} y^{1+\lambda}(s) d s \quad \text { for all } \quad t \in[0, T]
$$

with positive numbers $y_{0}, c_{0}$ and $\lambda$. Then

$$
y(t) \leq y_{0} \cdot\left(1-\lambda y_{0}^{\lambda} c_{0} t\right)^{-\frac{1}{\lambda}}
$$

holds for all $t \in[0, T]$ satisfying $t<\frac{1}{\lambda y_{0}^{\lambda} c_{0}}$.

Proof. The assertion will follow as soon as we have shown that for all $\varepsilon>0$ and each $t \in[0, T]$,

$$
y(t)<\left(y_{0}+\varepsilon\right) \cdot\left(1-\lambda\left(y_{0}+\varepsilon\right)^{\lambda} c_{0} t\right)^{-\frac{1}{\lambda}}=: y_{\varepsilon}(t) .
$$

Indeed, for $t=0$ this is obvious, hence if (2.9) was false there were $t_{0} \in(0, T]$ such that $y(t)<y_{\varepsilon}(t)$ for all $t<t_{0}$ and $y\left(t_{0}\right)=y_{\varepsilon}\left(t_{0}\right)$. Noting that $y_{\varepsilon}^{\prime}=c_{0} y_{\varepsilon}^{1+\lambda}$, 
we thus obtain

$$
\begin{aligned}
y_{\varepsilon}\left(t_{0}\right) & =y_{0}+\varepsilon+c_{0} \int_{0}^{t_{0}} y_{\varepsilon}(s)^{1+\lambda} d s \\
& >y_{0}+\varepsilon+c_{0} \int_{0}^{t_{0}} y(s)^{1+\lambda} d s \\
& >y\left(t_{0}\right),
\end{aligned}
$$

a contradiction.

We are now ready for the proof of Lemma 2.6.

Proof of Lemma 2.6. In view of the comparison principle, we may assume that $u$ is radially symmetric and nondecreasing with respect to $|x|$. With a small number $\gamma \in$ $(0,1)$ to be fixed below, we multiply $(2.1)$ by $u_{R j}^{-\gamma-1}$ and integrate over $B_{R} \times(0, t)$ to obtain

$$
\begin{array}{r}
\frac{1}{\gamma} \int_{B_{R}} u_{R j}^{-\gamma}(\cdot, t)+m(\gamma+1) \int_{0}^{t} \int_{B_{R}} u_{R j}^{m-\gamma-3}\left|\nabla u_{R j}\right|^{2}+m \int_{0}^{t} \int_{\partial B_{R}} u_{R j}^{m-\gamma-2} \partial_{\nu} u_{R j} \\
=\frac{1}{\gamma} \int_{B_{R}} u_{0 R j}^{-\gamma}+\int_{0}^{t} \int_{B_{R}} u_{R j}^{\kappa-\gamma-1} .
\end{array}
$$

From a simple comparison argument we gain $u_{R j} \leq j$ and therefore, since $\left.u_{R j}\right|_{\partial B_{R}}=j$, the third term on the left is nonnegative, while

$$
\begin{aligned}
m(\gamma+1) \int_{0}^{t} \int_{B_{R}} u_{R j}^{m-\gamma-3}\left|\nabla u_{R j}\right|^{2} & =\frac{4 m(\gamma+1)}{(1+\gamma-m)^{2}} \int_{0}^{t} \int_{B_{R}}\left|\nabla u_{R j}^{-\frac{1+\gamma-m}{2}}\right|^{2} \\
& \geq \frac{4 m}{(2-m)^{2}} \int_{0}^{t} \int_{B_{R}}\left|\nabla u_{R j}^{-\frac{1+\gamma-m}{2}}\right|^{2}
\end{aligned}
$$

because $\gamma \in(0,1)$. As $u_{R j} \nearrow u_{R}$ and $\nabla u_{R j} \rightarrow \nabla u_{R}$ a.e. in $B_{R} \times(0, t)$, Fatou's lemma and the monotone convergence theorem yield

$$
\begin{aligned}
\frac{1}{\gamma} \int_{B_{R}} u_{R}^{-\gamma}(\cdot, t)+\frac{4 m}{(2-m)^{2}} \int_{0}^{t} \int_{B_{R}}\left|\nabla u_{R}^{-\frac{1+\gamma-m}{2}}\right|^{2} \\
\leq \frac{1}{\gamma} \int_{B_{R}} u_{0 R}^{-\gamma}+\int_{0}^{t} \int_{B_{R}} u_{R}^{\kappa-\gamma-1} .
\end{aligned}
$$

Reformulated in terms of $v:=u_{R}^{-\frac{1+\gamma-m}{2}}$, this means that

$$
\begin{aligned}
\frac{1}{\gamma}\|v(\cdot, t)\|_{L^{\xi}\left(B_{R}\right)}^{\xi}+\frac{4 m}{(2-m)^{2}} & \int_{0}^{t}\|\nabla v(\cdot, s)\|_{L^{2}\left(B_{R}\right)}^{2} d s \\
& \leq \frac{1}{\gamma}\|v(\cdot, 0)\|_{L^{\xi}\left(B_{R}\right)}^{\xi}+\int_{0}^{t}\|v(\cdot, s)\|_{L^{\delta}\left(B_{R}\right)}^{\delta} d s,
\end{aligned}
$$


where $\xi:=\frac{2 \gamma}{1+\gamma-m}>0$ and $\delta:=\frac{2(1+\gamma-\kappa)}{1+\gamma-m}$ is smaller than 2 due to $\kappa>m$. Therefore the Gagliardo-Nirenberg inequality (2.4) gives

$$
\|v(\cdot, s)\|_{L^{\delta}\left(B_{R}\right)}^{\delta} \leq C_{G N}^{\delta}\|\nabla v(\cdot, s)\|_{L^{2}\left(B_{R}\right)}^{a \delta}\|v(\cdot, s)\|_{L^{\kappa}\left(B_{R}\right)}^{(1-a) \delta},
$$

with $C_{G N}$ independent of $R$ and $\gamma \leq 1$, where

$$
a=\frac{\frac{1}{\xi}-\frac{1}{\delta}}{\frac{1}{n}-\frac{1}{2}+\frac{1}{\xi}} .
$$

Since $a \delta=\frac{2 n(1-\kappa)}{2 \gamma+n-n m} \rightarrow \frac{2(1-\kappa)}{1-m}<2$ as $\gamma \rightarrow 0$, there exists some small $\gamma_{0}>0$ such that for any $\gamma \leq \gamma_{0}, a \delta \leq c_{2}$ with some constant $c_{2}<2$. Hence, if we employ Young's inequality in the form

$$
\begin{aligned}
A B \leq \eta A^{\theta}+c(\theta, \eta) B^{\frac{1}{1-\frac{1}{\theta}}}, \quad \text { for all } \quad A, B>0, \\
\text { where } c(\theta, \eta):=\frac{\theta-1}{\theta}(\theta \eta)^{-\frac{1}{\theta-1}},
\end{aligned}
$$

with $\eta:=\frac{4 m}{(2-m)^{2} C_{G N}^{\delta}}$ and $\theta:=\frac{2}{a \delta}>1$ then $c(r, \eta) \leq C$ for all $\gamma \leq \gamma_{0}$ and some $C>0$. Consequently, for such $\gamma$ we have

$$
\|v(\cdot, s)\|_{L^{\delta}\left(B_{R}\right)}^{\delta} \leq \frac{4 m}{(2-m)^{2}}\|\nabla v(\cdot, s)\|_{L^{2}\left(B_{R}\right)}^{2}+C\left(\|v(\cdot, s)\|_{L^{\xi}\left(B_{R}\right)}\right)^{\frac{(1-a) \delta}{1-\frac{a \delta}{2}}} .
$$

Inserting this into (2.12), for $y(t):=\|v(\cdot, t)\|_{L^{\xi}\left(B_{R}\right)}^{\xi}, \gamma \leq \gamma_{0}$, we obtain the inequality

$$
y(t) \leq y(0)+C \gamma \int_{0}^{t} y^{1+\lambda(\gamma)}(s) d s
$$

with

$$
\begin{aligned}
\lambda(\gamma) & :=\frac{(1-a) \delta}{1-\frac{a \delta}{2}} \frac{1}{\xi}-1 \\
& =\frac{2(1-\kappa)}{2 \gamma+n(\kappa-m)} \\
\nearrow \lambda_{0} & :=\frac{2(1-\kappa)}{n(\kappa-m)} \text { as } \gamma \searrow 0,
\end{aligned}
$$

where the first identity is verified by an elementary calculation. Thus, Lemma 2.8 entails

$$
y(t) \leq y(0)\left(1-\lambda(\gamma) y^{\lambda(\gamma)}(0) \cdot C \gamma t\right)^{-\frac{1}{\lambda(\gamma)}}
$$

for all $t>0$ satisfying $t<\frac{1}{\lambda(\gamma) y^{\lambda(\gamma)}(0) \cdot C \gamma}$. Now let $\tau>0$ be such that $u$ (and hence $u_{R} \geq u$ ) is strictly positive for $t \leq \tau$, which is possible due to Corollary 2.3, and let $c_{1}, M>1$ and $\mu<\mu_{0} \equiv \frac{1}{\lambda_{0}}$ denote the constants provided by Lemma 2.5 and hypothesis $(\mathrm{H})$, respectively. We fix $T>\tau$ and note that in order to prove (2.3) at 
time $t=T$ it is sufficient to consider the case that $u(0, T) \leq 1$. Given $\varepsilon \in\left(0, \frac{1}{\mu}\right)$, we pick an arbitrary $\gamma_{1}(\varepsilon)$ such that $\lambda(\gamma)>\lambda_{0}-\varepsilon$ for all $\gamma<\gamma_{1}(\varepsilon)$ and define

$$
\gamma \equiv \gamma(T):=\min \left\{1, \gamma_{0}, \gamma_{1}(\varepsilon),\left(2 C \lambda_{0} M^{\lambda_{0}} T\right)^{-\frac{1}{1-\lambda_{0} \mu}}\right\} .
$$

This guarantees that

$$
\lambda(\gamma) M^{\lambda(\gamma)} \gamma^{1-\lambda(\gamma) \mu} \cdot C T \leq \lambda_{0} M^{\lambda_{0}} \gamma^{1-\lambda_{0} \mu} \cdot C T \leq \frac{1}{2}
$$

and hence from (2.17) and (H) in the limit $R \rightarrow \infty$ we infer that

$$
\begin{aligned}
\int_{\mathbb{R}^{n}} u^{-\gamma}(\cdot, T) & \leq\left(\int_{\mathbb{R}^{n}} u_{0}^{-\gamma}\right) \cdot\left(1-\lambda(\gamma)\left(\int_{\mathbb{R}^{n}} u_{0}^{-\gamma}\right)^{\lambda(\gamma)} \cdot C \gamma T\right)^{-\frac{1}{\lambda(\gamma)}} \\
& \leq M \gamma^{-\mu} \cdot\left(1-\lambda(\gamma) M^{\lambda(\gamma)} \gamma^{-\lambda(\gamma) \mu} \cdot C \gamma T\right)^{-\frac{1}{\lambda(\gamma)}} \\
& \leq M \gamma^{-\mu} \cdot\left(\lambda_{0} M^{\lambda_{0}} \gamma^{1-\lambda_{0} \mu} \cdot C T\right)^{-\frac{1}{\lambda_{0}-\varepsilon}} \\
& =M^{-\frac{\varepsilon}{\lambda_{0}-\varepsilon}} \cdot\left(\lambda_{0} C\right)^{-\frac{1}{\lambda_{0}-\varepsilon}} \cdot \gamma^{-\frac{1-\varepsilon \mu}{\lambda_{0}-\varepsilon}} \cdot T^{-\frac{1}{\lambda_{0}-\varepsilon}} .
\end{aligned}
$$

On the other hand, by Lemma 2.5 , since $u(0, T) \leq 1$ and $\gamma \leq 1$, we have

$$
\begin{aligned}
\int_{\mathbb{R}^{n}} u^{-\gamma}(\cdot, T) & \geq(2 u(0, T))^{-\gamma} \cdot\left|\left\{x \in \mathbb{R}^{n}|| x \mid \leq c_{1} u^{-\frac{\kappa-m}{2 m}}(0, T)\right\}\right| \\
& \geq \frac{\omega_{n}}{2 n} c_{1}^{n} \cdot u^{-\frac{n(\kappa-m)}{2 m}}(0, T),
\end{aligned}
$$

where $\omega_{n}$ denotes the area of the unit sphere in $\mathbb{R}^{n}$. Combining (2.19) with (2.20), we end up with

$$
u(0, T) \geq\left(\frac{\omega_{n}}{2 n} c_{1}^{n} M^{\frac{\varepsilon}{\lambda_{0}-\varepsilon}}\left(\lambda_{0} C\right)^{\frac{1}{\lambda_{0}-\varepsilon}}\right)^{\frac{2 m}{n(\kappa-m)}} \cdot\left(\gamma^{\frac{1-\varepsilon \mu}{\lambda^{2}-\varepsilon}}(T) \cdot T^{\frac{1}{\lambda_{0}-\varepsilon}}\right)^{\frac{2 m}{n(\kappa-m)}} .
$$

Since the radial function $u(\cdot, T)$ is nondecreasing with respect to $|x|$, this shows that $u(\cdot, T)$ is positive in $\mathbb{R}^{n}$ for all $T>\tau$; by the choice of $\tau, u$ is thereby proved to be positive in $\mathbb{R}^{n} \times[0, \infty)$. Moreover, the definition $(2.18)$ of $\gamma(T)$ entails that with some constant $c_{3}>0$, for all $T>\tau$ we have $\gamma(T) \geq c_{3} T^{-\frac{1}{1-\lambda_{0} \mu}}$, and hence from (2.21) and the fact that $\mu_{0}=\frac{1}{\lambda_{0}}=\frac{n(\kappa-m)}{2(1-\kappa)}$ we obtain

$$
\begin{aligned}
\inf _{x \in \mathbb{R}^{n}} u(x, T) & \geq c_{4}\left(T^{-\frac{1}{1-\lambda_{0} \mu} \cdot \frac{1-\varepsilon \mu}{\lambda_{0}-\varepsilon}+\frac{1}{\lambda_{0}-\varepsilon}}\right)^{\frac{2 m}{n(\kappa-m)}} \\
& =c_{4} T^{-\frac{\mu \mu_{0}}{\mu_{0}-\mu} \cdot \frac{2 m}{n(\kappa-m)}} \\
& =c_{4} T^{-\frac{\mu m}{\left(\mu_{0}-\mu\right)(1-\kappa)}} \text { for all } T>0
\end{aligned}
$$

with a positive constant $c_{4}$. This proves (2.3).

Now that we have ruled out quenching in finite time, all that remains to show is that $u$ indeed quenches in infinite time. In fact, we can prove a somewhat sharper result. 
Theorem 2.9. Suppose $m \in(0,1), \kappa \in(m, 1)$, and assume that $u_{0}$ satisfies the growth assumption $(\mathrm{H})$. Then the solution $u$ constructed in Corollary 2.3 quenches regularly at $t=\infty$ in the sense of Definition 1.1. More precisely, for all compact $K \subset \subset \mathbb{R}^{n}$, we have

$$
\sup _{x \in K} u(x, t) \rightarrow 0 \quad \text { as } \quad t \rightarrow \infty,
$$

and there exists $c>0$ such that

$$
\inf _{x \in \mathbb{R}^{n}} u(x, t) \geq c t^{-\frac{\mu m}{\left(\mu_{0}-\mu\right)(1-\kappa)}}
$$

with $\mu$ and $\mu_{0}$ as specified in $(\mathrm{H})$.

Proof. The estimate (2.23) has just been proved in Lemma 2.6. To check (2.22), for given $K \subset \subset \mathbb{R}^{n}$ and $\varepsilon>0$, we fix some large $R>0$ such that $K \subset B_{\frac{R}{2}}$ and $R>\frac{4}{3} c_{0} \cdot\left(\frac{2}{\varepsilon}\right)^{\frac{1}{\alpha}}$, where again $\alpha:=\frac{2}{\kappa-m}$ and $c_{0}:=\sqrt{4 m \alpha(2 m \alpha+2+n)}$. By Corollary 2.3, $u$ is bounded, say, $u \leq c_{1}$, in $B_{R} \times(0, \infty)$, whence we may choose some positive $\delta<c_{0} c_{1}^{-\frac{1}{\alpha}} R$. Next, we let $y:[0, \infty) \rightarrow[0, \infty)$ be a continuously differentiable function with $y(0) \geq c_{1} R^{2 \alpha}$ which satisfies

$$
0 \geq y^{\prime} \geq-\frac{1}{2} \delta^{(1-\kappa) \alpha} y^{\kappa} \quad \text { in } \quad(0, \infty)
$$

as well as

$$
y(t) \searrow y_{\infty}:=c_{0}^{\alpha} R^{\alpha} \quad \text { as } \quad t \rightarrow \infty .
$$

Then

$$
v(x, t):=y(t) \cdot\left(R^{2}-|x|^{2}\right)^{-\alpha}, \quad(x, t) \in B_{\sqrt{R^{2}-\delta}} \times(0, \infty),
$$

fulfills

$$
\begin{aligned}
v(x, 0) & =y(0) \cdot\left(R^{2}-|x|^{2}\right)^{-\alpha} \\
& \geq y(0) \cdot R^{-2 \alpha} \\
& \geq c_{1} \\
& \geq u_{0}(x) \text { for all } x \in B_{\sqrt{R^{2}-\delta}},
\end{aligned}
$$

while for $x \in \partial B_{\sqrt{R^{2}-\delta}}$ and all $t>0$,

$$
\begin{aligned}
v(x, t) & =y(t) \cdot \delta^{-\alpha} \\
& \geq y_{\infty} \cdot \delta^{-\alpha} \\
& \geq c_{1} \\
& \geq u(x, t) .
\end{aligned}
$$

Moreover, using the definitions of $c_{0}$ and $\alpha$ and the properties of $y$ we find

$$
\begin{aligned}
& v_{t}-\Delta v^{m}+v^{\kappa} \\
&= y^{\prime} \cdot\left(R^{2}-|x|^{2}\right)^{-\alpha} \\
&-2 m \alpha\left[n\left(R^{2}-|x|^{2}\right)+2(m \alpha+1)|x|^{2}\right] y^{m}\left(R^{2}-|x|^{2}\right)^{-m \alpha-2} \\
&+y^{\kappa}\left(R^{2}-|x|^{2}\right)^{-\kappa \alpha}
\end{aligned}
$$




$$
\begin{aligned}
\geq & y^{\prime}\left(R^{2}-|x|^{2}\right)^{-\alpha} \\
& -\frac{1}{2} c_{0}^{2} R^{2} \cdot y^{m}\left(R^{2}-|x|^{2}\right)^{-m \alpha-2} \\
& +y^{\kappa}\left(R^{2}-|x|^{2}\right)^{-\kappa \alpha} \\
= & \left\{y^{\prime}+y^{\kappa}\left(R^{2}-|x|^{2}\right)^{(1-\kappa) \alpha} \cdot\left[1-\frac{1}{2} c_{0}^{2} R^{2} y^{-(\kappa-m)}\right]\right\} \cdot\left(R^{2}-|x|^{2}\right)^{-\alpha} \\
\geq & \left\{y^{\prime}+y^{\kappa}\left(R^{2}-|x|^{2}\right)^{(1-\kappa) \alpha} \cdot\left[1-\frac{1}{2} c_{0}^{2} R^{2} y_{\infty}^{-(\kappa-m)}\right]\right\} \cdot\left(R^{2}-|x|^{2}\right)^{-\alpha} \\
= & \left.\left\{y^{\prime}+y^{\kappa}\left(R^{2}-|x|^{2}\right)^{(1-\kappa) \alpha} \cdot \frac{1}{2}\right\} \cdot\left(R^{2}-|x|^{2}\right)^{-\alpha}\right] \\
\geq & \left\{y^{\prime}+\frac{1}{2} \delta^{(1-\kappa) \alpha} y^{\kappa}\right\} \cdot\left(R^{2}-|x|^{2}\right)^{-\alpha} \\
\geq & 0 \text { in } B \sqrt{R^{2}-\delta} \times(0, \infty),
\end{aligned}
$$

whereby it follows that $v \geq u$ in $B_{\sqrt{R^{2}-\delta}} \times(0, \infty)$. Thus, with $t_{0}$ large such that $y(t) \leq 2 y_{\infty}$ for $t \geq t_{0}$, we find that for any $x \in K$ and all $t \geq t_{0}$,

$$
\begin{aligned}
u(x, t) & \leq v(x, t) \\
& \leq 2 y_{\infty} \cdot\left(R^{2}-\left(\frac{R}{2}\right)^{2}\right)^{-\alpha} \\
& =2 \cdot\left(\frac{4}{3}\right)^{\alpha} c_{0}^{\alpha} R^{-\alpha} \\
& <\varepsilon
\end{aligned}
$$

according to our choice of $R$ and $y_{\infty}$. This proves the claim.

\section{Absence of regular infinite-time quenching for $\kappa<-m$}

Throughout this section, we assume that $n=1$, and that $u$ is a classical (and hence positive) solution of $(1.1)$ in $\mathbb{R} \times(0, \infty)$, satisfying $(1.2)$, and that

$u$ is symmetric with respect to $x=0$

$$
\text { and nondecreasing in } x \text { for } x \geq 0 \text { and } t \geq 0 \text {. }
$$

Our goal is to show that under these hypotheses, regular quenching in infinite time in the sense of Definition 1.1 does not occur when the absorption term in (1.1) is sufficiently strong, namely, if $\kappa<-m$. As a first step towards this, let us make sure that such type of singularity formation, if at all, can occur only at $x=0$. Note that this result is valid even for all $\kappa<0$.

Lemma 3.1. Suppose that $n=1, m \in(0,1)$ and $\kappa<0$, and assume that $u$ is a global classical solution of (1.1) satisfying (3.1) and (1.2). Then for all $x_{0}>0$ 
there exists $c\left(x_{0}\right)>0$ such that

$$
u\left(x_{0}, t\right) \geq c\left(x_{0}\right) \text { for all } t \geq 0 .
$$

Proof. Suppose on the contrary that for some $x_{0}>0$, there exists a sequence of times $t_{k} \rightarrow \infty$ such that $u\left(x_{0}, t_{k}\right) \rightarrow 0$ as $k \rightarrow \infty$. Then according to (3.1), we also have

$$
u\left(\cdot, t_{k}\right) \rightarrow 0 \text { uniformly in } \Omega:=\left(-x_{0}, x_{0}\right)
$$

as $k \rightarrow \infty$. Let $\Theta_{1}(x):=\frac{\pi}{4 x_{0}} \cos \frac{\pi x}{2 x_{0}}$ denote the principal Dirichlet eigenfunction of $-(\cdot)_{x x}$ in $\Omega$, which has been normalized such that $\int_{\Omega} \Theta_{1}(x) d x=1$. Multiplying (1.1) by $\Theta_{1}(x)$ and integrating over $\Omega$ yields

$$
\begin{aligned}
\frac{d}{d t} \int_{\Omega} u \Theta_{1} & =\int_{\Omega}\left(u^{m}\right)_{x x} \Theta_{1}-\int_{\Omega} u^{\kappa} \Theta_{1} \\
& =-\frac{\pi^{2}}{4 x_{0}^{2}} \int_{\Omega} u^{m} \Theta_{1}-\int_{\partial \Omega} u^{m} \partial_{\nu} \Theta_{1}-\int_{\Omega} u^{\kappa} \Theta_{1} \\
& \leq\left(C\left(\left[-x_{0}, x_{0}\right]\right)\right)^{m} \cdot \frac{\pi^{2}}{4 x_{0}^{2}}-\int_{\Omega} u^{\kappa} \Theta_{1},
\end{aligned}
$$

with $C\left(\left[-x_{0}, x_{0}\right]\right)$ taken from (1.2). Since $\kappa<0$, Hölder's inequality says that

$$
\int_{\Omega} u^{\kappa} \Theta_{1} \geq\left(\int_{\Omega} u \Theta_{1}\right)^{\kappa}
$$

so that from (3.4) we obtain that $y(t):=\int_{\Omega} u(x, t) \Theta_{1}(x) d x$ satisfies the singular ODI

$$
y^{\prime}(t) \leq c_{1}-y^{-\alpha}(t) \text { for all } t>0
$$

with a positive constant $c_{1}$ and $\alpha=-\kappa>0$. By (3.3), however, we know that $y\left(t_{k}\right) \rightarrow 0$ as $k \rightarrow \infty$, which along with an ODE comparison argument shows that $y$ must vanish at some finite time. This contradicts the assumed global positivity of $u$ and thereby finishes the proof.

One important feature of the parameter regime $\kappa<-m$ is the nonexistence of singular steady states - that is, of equilibria with zeroes. Regular stationary solutions, when known to be small at some point, therefore necessarily must become large even near this point, as will be shown in the following lemma. Observe that classical steady states of (1.1) are exactly the $\frac{1}{m}$-th powers of solutions $w$ of the ODE $w_{x x}=w^{-\beta-1}$ with $\beta:=\frac{-\kappa}{m}-1$ which is positive if $\kappa<-m$.

Lemma 3.2. Let $\beta>0$. For $\delta \in(0,1)$, consider the solutions of the initial-value problem

$$
\left\{\begin{array}{l}
w_{\delta x x}=w_{\delta}^{-\beta-1}, \quad x \in \mathbb{R} \\
w_{\delta}(0)=\delta, \quad w_{\delta x}(0)=0 .
\end{array}\right.
$$

Then for all $x \neq 0$ we have

$$
w_{\delta}(x) \rightarrow \infty \quad \text { as } \quad \delta \searrow 0 .
$$


Proof. Multiplying (3.5) by $w_{\delta x}$ and integrating twice, we obtain that $w_{\delta}$ is implicitly given by

$$
\int_{\delta}^{w_{\delta}(x)} \frac{d s}{\sqrt{\delta^{-\beta}-s^{-\beta}}}=\sqrt{\frac{2}{\beta}}|x|, \quad x \in \mathbb{R} .
$$

Substituting $s=\delta \sigma$, we find

$$
\delta^{1+\frac{\beta}{2}} \int_{1}^{\frac{w_{\delta}(x)}{\delta}} \frac{d \sigma}{\sqrt{1-\sigma^{-\beta}}}=\sqrt{\frac{2}{\beta}}|x|, \quad x \in \mathbb{R},
$$

from which it follows that

$$
\frac{w_{\delta}(x)}{\delta} \rightarrow \infty \quad \text { as } \quad \delta \searrow 0
$$

for each $x \neq 0$. Now for $z>2$ we have

$$
\begin{aligned}
\int_{1}^{z} \frac{d \sigma}{\sqrt{1-\sigma^{-\beta}}} & \leq \int_{1}^{2} \frac{d \sigma}{\sqrt{1-\sigma^{-\beta}}}+\int_{2}^{z} \frac{d \sigma}{\sqrt{1-2^{-\beta}}} \\
& =a+b z
\end{aligned}
$$

with the constants

$$
a=\int_{1}^{2} \frac{d \sigma}{\sqrt{1-\sigma^{-\beta}}}-\frac{2}{\sqrt{1-2^{-\beta}}} \quad \text { and } \quad b=\frac{1}{\sqrt{1-2^{-\beta}}} .
$$

For $x \neq 0$ and sufficiently small $\delta>0,(3.7)$ therefore yields the estimate

$$
\begin{aligned}
\sqrt{\frac{2}{\beta}}|x| & \leq \delta^{1+\frac{\beta}{2}}\left(a+b \cdot \frac{w_{\delta}(x)}{\delta}\right) \\
& =a \delta^{1+\frac{\beta}{2}}+b \delta^{\frac{\beta}{2}} w_{\delta}(x),
\end{aligned}
$$

which immediately implies (3.6), because $\beta>0$.

We proceed to derive from the lower pointwise estimate from Lemma 3.1 a statement that may be read as a weak type of upper estimate for quenching solutions.

Lemma 3.3. Assume $n=1, m \in(0,1), \kappa<-m$, and that $u$ is a global classical solution of (1.1) satisfying (3.1) and (1.2). If $u$ quenches at $t=\infty$ then for all $x_{0}>0$ we have

$$
\lim _{t \rightarrow \infty} \int_{-x_{0}}^{x_{0}} u^{-\lambda}(x, t) d x=\infty \quad \text { for all } \quad \lambda>\frac{m-\kappa}{2} .
$$

Proof. For convenience in notation, we transform (1.1) via the substitution $v(x, t)=u^{m}\left(x, \frac{t}{m}\right)$ into the problem

$$
\left\{\begin{array}{l}
v_{t}=v^{-p} v_{x x}-v^{-q}, \quad x \in \mathbb{R}, \quad t>0, \\
v(x, 0)=v_{0}(x):=u_{0}^{m}(x), \quad x \in \mathbb{R}
\end{array}\right.
$$


with new parameters $p=\frac{1-m}{m}>0$ and $q=\frac{1-m-\kappa}{m}$. Note that our assumption $\kappa<-m$ corresponds to the inequality $q>p+1$, and if $u$ quenches in infinite time then so does $v$.

Replacing $t$ with $\tilde{t}:=t+1$ if necessary, we may assume that $v_{0}$ is smooth on $\mathbb{R}$. Given $x_{0}>0$, we write $\Omega:=\left(-x_{0}, x_{0}\right)$. Since $v$ quenches at $t=\infty$, there exists a sequence of times $t_{k} \rightarrow \infty$ such that

$$
m_{k}:=v\left(0, t_{k}\right)=\min _{(x, t) \in \bar{\Omega} \times\left[0, t_{k}\right]} v(x, t) \quad \text { for all } \quad k \in \mathbb{N}
$$

and

$$
m_{k} \rightarrow 0 \quad \text { as } \quad k \rightarrow \infty
$$

For fixed $k \in \mathbb{N}$ we set

$$
P(x, t):=\frac{1}{2} v_{x}^{2}-f(v)-\eta t \quad \text { for } \quad(x, t) \in \bar{\Omega} \times\left[0, t_{k}\right] .
$$

Here, $\eta \in(0,1)$ is small and

$$
f(s):=\int_{m_{k}}^{s} \sigma^{-(q-p)} d \sigma=\frac{m_{k}^{-(q-p-1)}-s^{-(q-p-1)}}{q-p-1} \quad \text { for } \quad s \geq m_{k} .
$$

Observe that the hypothesis $q>p+1$ guarantees that $\int_{0}^{s} \sigma^{-(q-p)} d \sigma=\infty$ for all $s>0$. Thus, since $m_{k} \rightarrow 0$ as $k \rightarrow \infty$, we may assume that $f(v)$ is large enough so as to satisfy

$$
\frac{1}{2} v_{0 x}^{2}(x)<f\left(v_{0}(0)\right) \text { for all } x \in \Omega
$$

and

$$
\frac{1}{2} v_{x}^{2}\left(x_{0}, t\right) \leq f\left(c^{m}\left(x_{0}\right)\right) \text { for all } t \in(0, \infty),
$$

where $c\left(x_{0}\right)$ is as provided by Lemma 3.1. In asserting (3.11) we tacitly make use of the fact that (3.2) in conjunction with (1.2) implies that $u_{x}$ and hence $v_{x}$ is bounded in $\left(\frac{1}{2} x_{0}, 2 x_{0}\right) \times(0, \infty)$ by parabolic Schauder estimates. As a consequence of (3.10) and (3.11),

$$
P<0 \text { at } t=0 \text { and at } x= \pm x_{0} .
$$

Furthermore, $P$ satisfies

$$
P_{t}=A(x, t) P_{x x}+B(x, t) P_{x}+C(x, t) \quad \text { in } \quad\left\{(x, t) \in \bar{\Omega} \times\left(0, t_{k}\right] \mid u_{x}(x, t) \neq 0\right\}
$$

with

$$
\begin{aligned}
& A(x, t)=v^{-p}, \\
& B(x, t)=\frac{-v^{-p} v_{x x}-v^{-p} f^{\prime}}{v_{x}}-p v^{-p-1} v_{x}
\end{aligned}
$$

and

$$
\begin{aligned}
C(x, t) & =-v^{-p}\left(f^{\prime}\right)^{2}+v^{-q} f^{\prime}+\left(v^{-p} f^{\prime \prime}-p v^{-p-1} f^{\prime}+q v^{-q-1}\right) v_{x}^{2}-\eta \\
& =-\eta \quad<0
\end{aligned}
$$


because $f^{\prime}(s)=s^{-(q-p)}$ and $f^{\prime \prime}(s)=-(q-p) s^{-(q-p+1)}$. Note that since $f(v) \geq 0$, we have $P \leq 0$ at the points where $B$ might become singular (that is, where $\left.v_{x}=0\right)$. Accordingly, if the maximum of $P$ over $\bar{\Omega} \times\left[0, t_{k}\right]$ was positive, it would be attained at some point in $\Omega \times\left(0, t_{k}\right]$ and we would have $P_{x}=0, P_{x x} \leq 0$ and $P_{t} \geq 0$ at this point. However, this means that we would have

$$
0 \leq P_{t}-A P_{x x}-B P_{x}=C=-\eta<0
$$

there, which is impossible. We conclude that $P \leq 0$ in $\bar{\Omega} \times\left[0, t_{k}\right]$ and consequently, in the limit $\eta \rightarrow 0$, we obtain that

$$
\left|v_{x}\left(x, t_{k}\right)\right| \leq \sqrt{\frac{2}{q-p-1}\left(m_{k}^{-(q-p-1)}-v^{-(q-p-1)}\left(x, t_{k}\right)\right)} \quad \text { for } \quad x \in \Omega .
$$

A relation similar to the above one, with inequality sign replaced by ' $=$ ', is satisfied by some regular stationary solution: Namely, recalling the definition of $w_{\delta}$ given in Lemma 3.2 with $\beta=\frac{-\kappa}{m}-1 \equiv q-p-1>0$, we find that $w_{\delta}$ satisfies

$$
w_{\delta x}(x)=\operatorname{sign}(x) \cdot \sqrt{\frac{2}{q-p-1}\left(\delta^{-(q-p-1)}-w_{\delta}^{-(q-p-1)}(x)\right)} \text { for } x \neq 0 .
$$

Hence, an ODE comparison involving (3.12) shows that

$$
v\left(x, t_{k}\right) \leq w_{m_{k}}(x) \text { for all } x \in \bar{\Omega}
$$

and all large $k \in \mathbb{N}$.

In order to suitably extend this estimate to times $t$ not contained in the particular sequence $\left(t_{k}\right)_{k \in \mathbb{N}}$, we use a parabolic comparison argument. For this purpose, let $C(\bar{\Omega})$ denote the constant provided by (1.2). Then Lemma 3.2 yields some $\delta_{0}<1$ such that for all $\delta<\delta_{0}$, the solutions $w_{\delta}$ of (3.5) satisfy $w_{\delta}\left( \pm x_{0}\right)>$ $(C(\bar{\Omega}))^{m}$ and hence

$$
w_{\delta}\left( \pm x_{0}\right)>v\left( \pm x_{0}, t\right) \text { for all } t \geq 0 .
$$

In particular, using (3.14) we see that if $k$ is sufficiently large then $w_{m_{k}} \geq v$ on the parabolic boundary of $\Omega \times\left(t_{k}, \infty\right)$. Therefore the comparison principle entails that for such $k$,

$$
v(x, t) \leq w_{m_{k}}(x) \text { for all } x \in \Omega \text { and } t>t_{k} .
$$

Now in view of the mean value theorem, (3.13) implies that for $x \neq 0$

$$
\left|w_{\delta x}(x)\right| \leq \sqrt{2} \delta^{-\frac{q-p}{2}} \sqrt{w_{\delta}(x)-\delta}
$$

and hence upon integration we find

$$
w_{\delta}(x) \leq \delta+\frac{1}{2} \delta^{-(q-p)} x^{2} \text { for all } \quad x \neq 0 .
$$

Thus,

$$
w_{\delta}(x) \leq 2 \delta
$$


holds for all $x \in \mathbb{R}$ which satisfy

$$
\frac{1}{2} \delta^{-(q-p)} x^{2} \leq \delta
$$

or, equivalently,

$$
|x| \leq \sqrt{2} \delta^{\frac{q-p+1}{2}} .
$$

Using (3.14), for $\Lambda>0$ we therefore obtain

$$
\begin{aligned}
\int_{\Omega} v^{-\Lambda}(x, t) d x & \geq \int_{|x| \leq \sqrt{2} m_{k}^{\frac{q-p+1}{2}}}\left(2 m_{k}\right)^{-\Lambda} \\
& \geq 2 \sqrt{2} m_{k}^{\frac{q-p+1}{2}} \cdot\left(2 m_{k}\right)^{-\Lambda}
\end{aligned}
$$

for all $t>t_{k}$ and sufficiently large $k \in \mathbb{N}$. Since $m_{k} \rightarrow 0$ as $k \rightarrow \infty$ by assumption, this shows that $\int_{\Omega} v^{-\Lambda}(x, t) d x \rightarrow \infty$ as $t \rightarrow \infty$ whenever $\Lambda>\frac{q-p+1}{2}$. Transforming back to $u$, we end up with (3.8).

We proceed to prove our final result that rules out the possibility of regular quenching in infinite time for $\kappa<-m$.

Theorem 3.4. Let $n=1$, and assume that $m \in(0,1)$ and $\kappa<-m$. Then there is no solution to (1.1) satisfying (3.1) and (1.2) that quenches in infinite time.

Proof. Suppose on the contrary that such a quenching solution exists. We then integrate (1.1) over $\Omega:=\left(-\frac{1}{2}, \frac{1}{2}\right)$ to obtain

$$
\frac{d}{d t} \int_{\Omega} u=\int_{\partial \Omega} \partial_{\nu} u^{m}-\int_{\Omega} u^{\kappa} \text { for all } t>0 .
$$

By (1.2) and Lemma 3.1, $u$ is bounded from above and below by positive constants in $G \times(0, \infty)$, where $G:=\left(-\frac{3}{4},-\frac{1}{4}\right) \cup\left(\frac{1}{4}, \frac{3}{4}\right)$, which means that (1.1) is uniformly parabolic there. As a consequence, interior Schauder estimates for quasilinear parabolic equations [18, Theorem V.3.1] provide a bound for $u_{x}$ in $C^{\theta, \frac{\theta}{2}}\left(G^{\prime} \times(1, \infty)\right)$ for some $\theta>0$, where $G^{\prime}:=\left(-\frac{5}{8},-\frac{3}{8}\right) \cup\left(\frac{3}{8}, \frac{5}{8}\right)$. In particular, we obtain

$$
\int_{\partial \Omega} \partial_{\nu} u^{m}(\cdot, t) \leq c \quad \forall t>1
$$

with some $c>0$, while Lemma 3.3 entails that

$$
\int_{\Omega} u^{\kappa}(\cdot, t) \geq 2 c \text { for all sufficiently large } t
$$

because $-\kappa>\frac{m-\kappa}{2}$ due to our assumption on $\kappa$. Using this in (3.17) and applying Hölder's inequality, for large $t$ we obtain

$$
\begin{aligned}
\frac{d}{d t} \int_{\Omega} u & \leq-\frac{1}{2} \int_{\Omega} u^{\kappa} \\
& \leq-\frac{1}{2}\left(\int_{\Omega} u\right)^{\kappa} .
\end{aligned}
$$


As $\kappa<0$, this means that $\int_{\Omega} u(x, t) d x$ must vanish at some finite time, contradicting the positivity of $u$.

\section{Acknowledgements}

The author would like to thank Marek Fila and Christian Stinner for fruitful discussions on the topic of this paper.

\section{References}

[1] D. G. Aronson: The porous medium equation. Nonlinear diffusion problems, Lect. 2nd 1985 Sess. C.I.M.E.. Montecatini Terme/Italy 1985, Lect. Notes Math. 1224, 1-46 (1986).

[2] M. Borelli, M. Ughi: The fast diffusion equation with strong absorption: The instantaneous shrinking phenomenon. Rend. Ist. Mat. Univ. Trieste 26, (1-2), 109-140 (1994).

[3] X.-Y. Chen, H. Matano, M. Mimura: Finite-point extinction and continuity of interfaces in a nonlinear diffusion equation with strong absorption. J. reine angew. Math. 459, 1-36 (1995).

[4] L. C. Evans, B. F. Knerr: Instantaneous shrinking of the support of nonnegative solutions to certain nonlinear parabolic equations and variational inequalities. Illinois J. of Math. 23 (1), 153-166 (1979).

[5] M. Fila, J. Hulshof, P. Quittner: The Quenching Problem on the N-dimensional Ball. Nonlinear diffusion equations and their equilibrium states, Proc. 3rd Conf., Gregynog/UK 1989, Prog. Nonlinear Differ. Equ. Appl. 7, 183-196 (1992).

[6] M. Fila, B. Kawohl: Is quenching in infinite time possible? Q. Appl. Math. 48 (3), 531-534 (1990).

[7] M. Fila, B. Kawohl: Asymptotic analysis of quenching problems. Rocky Mt. J. Math. 22 (2), 563-577 (1992).

[8] M. Fila, King, J. R., M. Winkler, E. Yanagida: Large time behaviour of solutions of a semilinear heat equation with supercritical nonlinearity. To appear in: Proceedings of 'Equadiff 2007', Wien 2008.

[9] M. Fila, Levine, H. A., Vázquez, J. L.: Stabilization of solutions of weakly singular quenching problems. Proc. Am. Math. Soc. 119 (2), 555-559 (1993).

[10] S. Filippas, J. S. Guo: Quenching profiles for one-dimensional semilinear heatq equations. Q. Appl. Math. 51, 713-729 (1993).

[11] A. Friedman, B. McLeod: Blow-up of positive solutions of semilinear heat equations. Indiana Univ. Math. J. 35, 425-447 (1985).

[12] V. A. Galaktionov, S. I. Shmarev, J. L. Vazquez: Regularity of interfaces in diffusion processes under the influence of strong absorption. Arch. Rat. Mech. Anal. 149 (3), 183-212 (1999).

[13] B. H. Gilding, R. Kersner: Instantaneous extinction, step discontinuities and blow-up. Nonlinearity 16, 843-854 (2003). 
[14] J.-S. Guo: On the quenching behavior of the solution of a semilinear parabolic equation. J. Math. Anal. Appl. 151, 58-79 (1990).

[15] J.-S. Guo, Ph. Souplet: Fast rate of formation of dead-core for the heat equation with strong absorption and applications to fast blow-up. Math. Ann. 331, 651-667 (2005).

[16] R. S. Hamilton: The Ricci flow on surfaces. Contemp. Math. 71, 237-262 (1988).

[17] S. Kaplan: On the growth of solutions of quasilinear parabolic equations. Comm. Pure Appl. Math 16, 327-330 (1963).

[18] O. A. Ladyzenskaja, V.A. Solonnikov, N. N. Ural'ceva, Linear and Quasi-linear Equations of Parabolic Type, AMS, Providence (1968).

[19] H. A. Levine: The phenomenon of quenching: a survey, in: Trends in the Theory and Practice of Nonlinear Analysis, V. Lakshmikantham (ed.) North Holland, 257-286 (1985).

[20] H. Tanabe: Functional analytical methods for partial differential equations. Dekker, New York (1997).

[21] M. Winkler: Quenching phenomena in strongly degenerate diffusion equations with strong absorption. Journal of Math. Analysis and Applications 288, 481-504 (2003).

[22] M. Winkler: Blow-up of solutions to a degenerate parabolic equation not in divergence form. J. Differ. Equations 192 (2), 445-474 (2003).

[23] M. Winkler: On strongly degenerate diffusion with strong absorption. Habilitation thesis, Aachen (2004).

Michael Winkler

Department of Applied Mathematics and Statistics

Comenius University

84248 Bratislava

Slovakia

e-mail: michael.winkler@uni-due.de

Received: 24 May 2008.

Accepted: 19 November 2008. 